\title{
CONTINUITY OF THE SPECTRUM AND SPECTRAL RADIUS
}

\author{
GERARD J. MURPHY
}

\begin{abstract}
Let $A$ be a Banach algebra containing an element $x$. Topological conditions on the spectrum of $x$ are given which are necessary and sufficient to ensure the continuity of the spectrum or spectral radius at $x$.
\end{abstract}

1. Introduction. In this paper $\mathbf{C}$ denotes the field of complex numbers, and $\mathbf{K}$ the metric space of nonempty compact subsets of $\mathbf{C}$ endowed with the Hausdorff metric $\Delta$. If $K_{1}, K_{2} \in \mathbf{K}$, then

$$
\Delta\left(K_{1}, K_{2}\right)=\max \left(\sup _{\lambda \in K_{2}} d\left(\lambda, K_{1}\right), \sup _{\lambda \in K_{1}} d\left(\lambda, K_{2}\right)\right)
$$

where $d\left(\lambda, K_{1}\right)=\inf _{\mu \in K_{1}}|\lambda-\mu|$.

$A$ will denote a Banach algebra, which we will always assume is unital without loss of generality. For $x \in A, \sigma(x)$ and $r(x)$ denote the spectrum of $x$ and spectral radius of $x$ respectively. We are interested in determining the points in $A$ at which the spectrum $\sigma: A \rightarrow \mathbf{K}, x \rightarrow \sigma(x)$ or the spectral radius $r: A \rightarrow[0, \infty), x \rightarrow r(x)$ are continuous. Newburgh [5] initiated the study of this problem in 1951. A famous example, due to Kakutani [4, pp. 248-249], shows that the spectral radius is discontinuous at certain elements in the $C^{*}$-algebra $B(H)$ of all bounded linear operators on a separable Hilbert space $H$. Recently Conway and Morrel [3] have given necessary and sufficient conditions for the continuity of $\sigma$ and $r$ at $T$ in $B(H)$. Their results depend on a deep theorem of Apostol and Morrel [1, Theorem 3.1] a special case of which we now state, as it will be used in the sequel.

THEOREM 1. If $T$ is a normal operator on $H$, and $S$ is a closed subset of $\mathbf{C}$ which meets all the components of $\sigma(T)$, then there is a sequence $T_{n}$ in $B(H)$ converging to $T$ in norm for which $\sigma\left(T_{n}\right) \subseteq S(n>0)$.

Let $A$ be a Banach algebra, $x \in A$, and $U$ an open subset of $C$. There are a number of results, due essentially to Newburgh [5] which we shall need later.

Theorem 2. If $U \supseteq \sigma(x)$, then there exists $\delta>0$ such that $\|y-x\|<\delta$ implies that $U \supseteq \sigma(y)$.

(This property of $\sigma$ is referred to as upper semicontinuity.)

Received by the editors June 18, 1980.

1980 Mathematics Subject Classification. Primary $46 \mathrm{HOS.}$

() 1981 American Mathematical Society 0002-9939/81/0000-0371/\$01.75 
THEOREM 3. If $U$ contains a component of $\sigma(x)$, then there exists $\delta>0$ such that $\|y-x\|<\delta$ implies that $U$ contains a component of $\sigma(y)$.

It is trivial to see that $r$ is always upper semicontinuous, and that, since $r(x)=\Delta(\sigma(x),\{0\})$, if $\sigma$ is continuous at $x$, so is $r$. An interesting result due to Aupetit [2] states that $r$ is uniformly continuous on $A$ if and only if $A / \operatorname{rad}(A)$ is commutative $(\operatorname{rad}(A)$ denotes the Jacobson radical of $A)$.

2. Continuity of $r$ and $\sigma$. Throughout this section $A$ is a (unital) Banach algebra, $x \in A$, and $T$ a normal operator on a separable infinite dimensional Hilbert space $H$. Let $K \in \mathbf{K}$, and put $\alpha(K)=\sup \left\{\inf _{\lambda \in \omega}|\lambda|: \omega\right.$ is a component of $\left.K\right\}$, and $r(K)=\sup _{\lambda \in K}|\lambda|$. So $r(K)>\alpha(K)$. We assume $\sigma(T)=K$.

Definition 1. The set $K$ is an $r$-set (resp. $\sigma$-set) if for every Banach algebra $A$, and $x$ in $A$ with $\sigma(x)=K$, the spectral radius $r$ (resp. the spectrum $\sigma$ ) is continuous at $x$ in $A$. A well-known result of Newburgh [5] can be restated by saying that if $K$ is totally disconnected it is a $\sigma$-set.

(Note that this is true for the spectrum $\sigma(S)$ of a compact or Riesz operator $S$ on a Banach space $X$, so that $\sigma$ is continuous at $S$ in the Banach algebra $B(X)$ of bounded linear operators on $X$.)

Assume $K$ is a member of $\mathbf{K}$.

Proposition 1. The following are equivalent statements:

(i) $K$ is an $r$-set;

(ii) $\alpha(K)=r(K)$;

(iii) $T$ is a point of continuity of $r$ in $B(H)$.

Proof. (ii) $\Rightarrow$ (i). If $\alpha(K)=r(K)$ and $K=\sigma(x)$ for $x$ in some Banach algebra $A$ then, for any $\varepsilon>0$, let $U=\{\lambda \in \mathbf{C}:|\lambda|>r(x)-\varepsilon\}$. As there is a component $\omega$ of $K$ with $\inf _{\lambda \in \omega}|\lambda|>\alpha(K)-\varepsilon=r(x)-\varepsilon$, so $U \supseteq \omega$, and hence by Theorem 3, there exists $\delta>0$ such that $\|y-x\|<\delta$ implies that $U$ contains a component of $\sigma(y)$. Therefore $r(y)>r(x)-\varepsilon$. This proves the lower semicontinuity of $r$ at $x$. As upper semicontinuity is automatic, it follows that $r$ is continuous at $x$. Thus we have shown that $K$ is an $r$-set.

(i) $\Rightarrow$ (iii) by definition.

(iii) $\Rightarrow$ (ii). Assume that (iii) holds and that $\alpha(K)<r(K)$. (We can always construct a normal $T$ with $\sigma(T)=K$, simply by choosing a diagonal operator whose diagonal entries are dense in $K$.) Now every component $\omega$ of $K$ meets the set $\Delta=\{\lambda \in \mathbf{C}:|\lambda| \leqslant \rho\}$ where $\rho$ is chosen such that $\alpha(K)<\rho<r(K)$. Hence by Theorem 1 , there is a sequence $T_{n}$ in $B(H)$ converging to $T$ in norm with $\sigma\left(T_{n}\right) \subseteq \Delta$. Thus $r\left(T_{n}\right) \leqslant \rho(n>0)$. But this is impossible by the continuity of $r$ at $T$. This contradiction shows $\alpha(K)=r(K)$, and so (iii) $\Rightarrow$ (ii). The proof of implication (iii) $\Rightarrow$ (ii) is a simplification of a special case of the proof of Theorem 2.6 in [3].

For $K \in \mathbf{K}$, let $K_{0}=\{\lambda \in K$ : the component of $\lambda$ in $K$ is $\{\lambda\}\}$. (Thus $K=K_{0}$ if and only if $K_{0}$ is totally disconnected.) As before, $T$ is a normal operator on $H$ and $\sigma(T)=K . \bar{K}_{0}$ denotes the closure of $K_{0}$ in $\mathbf{C}$. 
Proposition 2. The following are equivalent statements:

(i) $K$ is a $\sigma-s e t$;

(ii) $K=\bar{K}_{0}$;

(iii) for each $\varepsilon>0$ and for each $\lambda \in K, B(\lambda, \varepsilon)=\{\mu \in \mathbf{C}:|\mu-\lambda|<\varepsilon\}$ contains a component of $K$;

(iv) $T$ is a point of continuity of $\sigma$ in $B(H)$.

Proof. That (iii) $\Rightarrow$ (ii) follows from some elementary topology. (This fact is also pointed out in [3, p. 19].)

(ii) $\Rightarrow$ (i). Let $A$ be any Banach algebra, and suppose that $\sigma(x)=K$. Then for each $\varepsilon>0$, there exist $\lambda_{1}, \ldots, \lambda_{n} \in K$ such that $B\left(\lambda_{1}, \varepsilon / 2\right) \cup \cdots \cup B\left(\lambda_{n}, \varepsilon / 2\right) \supseteq$ $K$. Hence as $\bar{K}_{0}=K$, there exist $\mu_{i} \in K_{0}(i=1, \ldots, n)$ such that $\left|\lambda_{i}-\mu_{i}\right|<\varepsilon / 2$. Thus there exist $\delta_{i}>0(i=1, \ldots, n)$ such that $\|y-x\|<\delta_{i}$ implies that $B\left(\lambda_{i}, \varepsilon / 2\right)$ contains a component of $\sigma(y)$ (this follows from Theorem 3, and the fact that $\left\{\mu_{i}\right\}$ is a component of $\left.\sigma(x)=K\right)$. Thus if $\delta=\min _{1<i<n} \delta_{i}$ then for $\|y-x\|$ $<\delta$ and $\lambda \in \sigma(x)$ we have $\left|\lambda-\lambda_{i}\right|<\varepsilon / 2$ for some $i, 1<i<n$, and, for each $i$, $\left|\lambda_{i}-\lambda_{i}^{\prime}\right|<\varepsilon / 2$ for some $\lambda_{i}^{\prime}$ in $\sigma(y)$, as $B\left(\lambda_{i}, \varepsilon / 2\right)$ contains a component of $\sigma(y)$.

Hence $\left|\lambda-\lambda_{i}^{\prime}\right|<\varepsilon$, or $d(\lambda, \sigma(y))<\varepsilon$. Thus $\sup _{\lambda \in \sigma(x)} d(\lambda, \sigma(y))<\varepsilon$ for $\|y-x\|$ $<\delta$.

But by the upper semicontinuity property, there exists $\delta_{0}<\delta, \delta_{0}>0$, such that $\|y-x\|<\delta_{0}$ implies that $\sup _{\lambda \in \sigma(y)} d(\lambda, \sigma(x))<\varepsilon$. Thus $\Delta(\sigma(y), \sigma(x))<\varepsilon$ for $\|y-x\|<\delta_{0}$, showing that $\sigma$ is continuous at $x$. Hence $K$ is a $\sigma$-set. (This argument is a generalization of one due to Newburgh [5] proving that totally disconnected sets are $\sigma$-sets.)

(i) $\Rightarrow$ (iv) by definition.

(iv) $\Rightarrow$ (iii). Assume that $T$ is a point of continuity of $\sigma$ and let $\varepsilon>0$ and $\lambda \in K=\sigma(T)$. If $B(\lambda, \varepsilon)$ does not contain a component of $K$, then $S=C \backslash B(\lambda, \varepsilon)$ is a closed set meeting all the components of $\sigma(T)$, so by Theorem 1 , there is a sequence $T_{n}$ in $B(H)$ with $T_{n}$ converging to $T$ in norm, but $\sigma\left(T_{n}\right) \subseteq S(n>0)$. Hence $\Delta\left(\sigma\left(T_{n}\right), \sigma(T)\right)>d\left(\lambda, \sigma\left(T_{n}\right)\right)>\varepsilon(n>0)$. But as $\sigma$ is continuous at $T$, $\Delta\left(\sigma\left(T_{n}\right), \sigma(T)\right) \rightarrow 0(n \rightarrow \infty)$. This contradiction shows $B(\lambda, \varepsilon)$ contains a component of $\sigma(T)$, and so (iv) implies (iii). (This result is a simplified proof of a special case of Theorem 3.1 in [3].)

Finally, it is easy to exhibit $K \in \mathbf{K}$ such that $K_{0} \neq K=\bar{K}_{0}$ and to exhibit $K$ such that $\alpha(K)=r(K)$ but no component of $K$ lies on the circle $\{\lambda:|\lambda|=r(K)\}$. (For example take $K=\{1-1 / n: n>1\} \cup\{x+i y: x+y=1 ; x, y>0\}$.)

\section{REFERENCES}

1. C. Apostol and B. Morrel, On uniform approximation of operators by simple models, Indiana Univ. Math. J. 26 (1977), 427-442.

2. B. Aupetit, Characterisation spectrale des algèbres de Banach commutatives, Pacific J. Math. 63 (1976), 23-35.

3. J. Conway and B. Morrel, Operators that are points of spectral contimuity, Integral Equations Operator Theory 2 (1979), 174-198.

4. P. Halmos, A Hilbert space problem book, Van Nostrand, Princeton, N.J., 1967.

5. J. Newburgh, The variation of spectra, Duke Math. J. 18 (1951), 165-176.

School of Mathematics, Trinity College, Dublin, Ireland

Current address: Department of Mathematics, Dalhousie University, Halifax, Nova Scotia, Canada 